\title{
Comparação de métodos de avaliação dos valores energéticos do grão de trigo e seus subprodutos para frangos de corte
}

[Evaluation methodology of energy values of wheat grain and its by-products for broiler chickens]

\author{
F.M.O. Borges ${ }^{1}$, H.S. Rostagno ${ }^{2}$, C.E.P. Saad ${ }^{3}$, N.M. Rodriguez ${ }^{4}$, E.A. Teixeira ${ }^{5}$, \\ L.B. Lara $^{5}$, W.S. Mendes ${ }^{5}$, V.L. Araújo \\ ${ }^{1}$ Departamento de Zootecnia da Universidade Federal de Lavras \\ Caixa Postal 37 \\ 37200-000 - Lavras, MG \\ ${ }^{2}$ Universidade Federal de Viçosa \\ ${ }^{3}$ Zootecnista \\ ${ }^{4}$ Escola de Veterinária da Universidade Federal de Minas Gerais \\ ${ }^{5}$ Médico Veterinário
}

Recebido para publicação em 13 de fevereiro de 2003

Recebido para publicação, após modificações, em 22 de setembro de 2003

E-mail: borgesvet@ufla.br

\section{RESUMO}

Realizou-se um experimento com frangos de corte para avaliação da energia metabolizável ((energia metabolizável aparente (EMA), energia metabolizável verdadeira (EMV), energia metabolizável aparente corrigida pelo nitrogênio $\left(\mathrm{EMA}_{\mathrm{n}}\right)$ e energia metabolizável verdadeira corrigida pelo nitrogênio $\left(\mathrm{EMV}_{\mathrm{n}}\right)$ ), e comparação das metodologias tradicional e de alimentação forçada, em delineamento experimental inteiramente ao acaso, em esquema fatorial $4 \times 8$ (métodos $\times$ alimentos) com quatro repetições. Sete alimentos oriundos do trigo e uma ração-referência foram utilizados nas quatro metodologias: trigo integral moído, gérmen de trigo, farinha de trigo clara, farinha de trigo escura, farelo de trigo para uso humano, farelo de trigo para uso animal e farelo de trigo grosso. Os métodos 1 e 2 foram baseados na metodologia tradicional de consumo à vontade, usando-se aves com 14 e 39 dias de idade, respectivamente. Os alimentos testados substituíram $40 \%$ de uma dieta-referência. Nas metodologias 3 e 4 utilizou-se o método da alimentação forçada. Neles as aves, submetidas a jejum de 30 horas, foram forçadas a ingerir 25 gramas de alimento de uma única vez, utilizando o alimento puro, sem níveis de substituição. Em todos os métodos houve um tratamento extra no qual todas as aves ficaram em jejum, para determinação das perdas metabólica e endógena. O método 4 foi realizado com aves previamente cecectomizadas. Nos alimentos e nas excretas foram analisadas matéria seca, proteína bruta e energia bruta para determinação de energia metabolizável. A EMA e a EMA $\mathrm{E}_{n}$ foram menores nos métodos com consumo forçado, e as aves cecectomizadas apresentaram os maiores valores de EMV. Quanto a $\mathrm{EMV}_{\mathrm{n}}$, pela metodologia tradicional não houve diferença entre tratamentos, entretanto alguns tratamentos dessa metodologia diferiram dos tratamentos de alimentação forçada. As relações EMV/EMA e $\mathrm{EMV}_{\mathrm{n}} / \mathrm{EMA}_{\mathrm{n}}$ mostraram-se mais estreitas nos métodos com consumo à vontade.

Palavras-chave: frango, energia metabolizável aparente, energia metabolizável verdadeira, balanço de nitrogênio

\section{ABSTRACT}

One experiment was run with broiler chickens to evaluate the metabolizable energy (ME), ((apparent (MEA), true (MEV), apparent corrected for nitrogen retention $\left(M E A_{n}\right)$ and true corrected for nitrogen retention $\left.\left(M E V_{n}\right)\right)$. The traditional and the forced fed methods were compared in a factorial design $4 \times 8$ 
(methods $\times$ feedstuffs) with four replicates. Seven feed ingredients originating from wheat were used in five experiments: wheat grain, wheat germ, white wheat flour, dark wheat flour, wheat bran for human use, wheat bran for animal use and rough wheat bran. The first two methods were the traditional methodology with ad libitum feeding, using birds with different ages, 13 and 39 day-old, respectively. The feedstuffs tested substituted $40 \%$ of a reference diet. In methods three and four the birds were fasted 30 hours and forced fed $25 \mathrm{~g}$ of the feed ingredients (Sibbald, 1976). In all the experiments a treatment was included with fasting birds, to determine metabolic and endogenous losses. In method four caecectomized birds were used. Feeds and excreta were analyzed for dry matter, protein and gross energy for determination of the metabolizable energy values. MEA and $M E A_{n}$ values were smaller when the birds were forced fed. Caecectomized birds showed the highest values of MEV. The feedstuffs $M E V_{n}$ values determined by the traditional method did not show statistical differences among the feeds, however some feed ingredients resulted in different values when compared with those determined with forced fed birds. The MEV/MEA and $M E V_{n} / M E A_{n}$ relationships were narrowed in the methods with ad libitum feed consumption.

Keywords: bird, apparent metabolizable energy, true metabolizable energy, nitrogen balance

\section{INTRODUÇÃO}

Segundo Albino et al. (1994), as necessidades energéticas das aves são expressas principalmente como energia metabolizável (EM), visto ser a melhor forma para estimar a energia disponível dos alimentos. A precisão desses valores está diretamente relacionada com a eficiência dos sistemas de produção, no que diz respeito à produtividade e rentabilidade.

A energia da excreta é composta de energia proveniente de uma fração não assimilada do alimento e de uma fração de origem endógena e independente da dieta. Quando essa última fração não é considerada nos cálculos tem-se a EM aparente (EMA), entretanto, quando considerada, tem-se a EM verdadeira (EMV). Nas últimas décadas a energia foi expressa principalmente sob a forma de EMA (Lima et al., 1989; Albino, 1991).

A metodologia tradicional de avaliação energética apresenta como característica primária à utilização de uma dieta basal administrada a um grupo de aves-controle, na qual um de seus constituintes é substituído pelo ingrediente a ser utilizado, além do consumo ser ad libitum. Segundo Schang (1987), esse procedimento assume que toda variação no resultado da EMA da dieta é devida ao ingrediente-teste, não levando em consideração o nível de inclusão e o valor extracalórico de alguns alimentos.

Assim, Sibbald (1976) sugeriu uma metodologia que considerasse a fração endógena e metabólica da dieta, e que consistia basicamente em alimentar forçadamente galos adultos em balanço de nitrogênio com pequenas quantidades dos alimentos a serem testados. Nela um grupo de aves é deixado em jejum, para obtenção das perdas metabólicas e endógenas. Segundo o autor, quando o nível de consumo é alto, a influência das perdas metabólicas é pequena, entretanto, quando o consumo é baixo, essas perdas podem diminuir consideravelmente a EMA. Além disso, o pesquisador justificou que a fração endógena que compõe a excreta das aves alimentadas é a mesma que a das aves em jejum.

Ao longo do tempo essa metodologia foi sofrendo modificações devido às sugestões de outros pesquisadores e observações do próprio autor. Uma modificação significativa foi a correção da EMV pelo balanço de nitrogênio (BN) (Sibbald, Morse, 1982; Sibbald, Wolynetz, 1985; Sibbald, Wolynetz, 1988), denominado energia metabolizável verdadeira corrigida $\left(E V_{\mathrm{n}}\right)$. Sibbald e Wolynetz (1985) afirmaram ser a $E M V_{n}$ a estimativa de maior precisão para o conteúdo energético dos alimentos, uma vez que independe dos níveis de ingestão.

A metodologia de alimentação forçada apresenta a vantagem de ser mais rápida e menos onerosa do que a metodologia tradicional. A principal dificuldade encontrada para se utilizar valores de EMV dos 
alimentos na formulação de ração para aves é que todos os padrões nutricionais são baseados em EMA e nem todos os alimentos têm seus valores de EMV conhecidos. A metodologia de alimentação forçada é realizada com aves adultas, entretanto sabe-se que a idade das aves pode influenciar os valores de EM, isto é, aves adultas aproveitam melhor a energia dos alimentos, particularmente os ricos em fibra. (Härtel, 1986).

O bioensaio proposto por Sibbald (1976) para estimativa da energia metabolizável verdadeira pode-se prestar à determinação dos aminoácidos digestíveis. Likuski e Dorell (1978) citam que esse método pode ser interessante uma vez que é rápido e de baixo custo. Além disso, pouco se sabe, segundo Hayes et al. (1990), a respeito da influência do ceco sobre a energia metabolizável.

Muramatsu et al. (1994) citaram que os efeitos da microflora intestinal no metabolismo da energia podem ser evidentes. Em um experimento no qual se variou a quantidade de energia fornecida (de zero a suficiente para a manutenção) para aves normais e livres de germe, os autores observaram que quando a energia dietética não era disponível, a presença da flora microbiana beneficiava as aves por diminuição das perdas endógenas. Quando a energia dietética era acima da manutenção, a eficiência de utilização energética era diminuída pela presença da microflora.

Em vista da dificuldade de se obter e trabalhar com aves "germ-free", pode-se trabalhar com aves cecectomizadas, isto é, aves que tiveram seus cecos extirpados cirurgicamente.

Em vista do exposto esse trabalho teve por objetivos: comparar a metodologia tradicional e a de alimentação forçada para a determinação dos valores de EMA, EMV, energia metabolizável aparente corrigida $\left(E M A_{n}\right)$ e $E M V_{n}$ do trigo e vários de seus subprodutos para aves, comparar os valores de energia metabolizável dos alimentos obtidos com pintos e frangos e comparar os valores de EMA, EMV, $\mathrm{EMA}_{\mathrm{n}}$ e $\mathrm{EMV}_{\mathrm{n}}$ do trigo e vários de seus subprodutos para aves cecectomizadas e aves intactas.

\section{MATERIAL E MÉTODOS}

Realizou-se um experimento para avaliar energia metabolizável do trigo e seus subprodutos para aves, utilizando-se quatro metodologias de avaliação. Uma ração-referência (RR) e sete alimentos-teste, constituídos de produtos oriundos do trigo, foram utilizados em todos os métodos: trigo integral moído (TIM), gérmen de trigo (GT), farinha de trigo clara (FTC), farinha de trigo escura (FTE), farelo de trigo para uso humano (FTH), farelo de trigo para uso animal (FTA) e farelo de trigo grosso (FTG).

Os alimentos testados apresentaram composição química bastante diferente.

No método 1 foram utilizados 360 pintos de corte, da linhagem "Hubbard", machos, com 14 dias de idade. Trezentos e vinte pintos foram distribuídos ao acaso em oito tratamentos, com quatro repetições de 10 aves cada. Os 40 pintos restantes foram empregados em um tratamento extra. Eles permaneceram em jejum por 72 horas para se avaliar as perdas endógena e metabólica, de modo a determinar um fator de correção para estimar a energia metabolizável verdadeira dos alimentos. Esse tratamento (9) não fez parte das análises estatísticas para a determinação da EM. Os pintos foram alojadas em gaiolas metálicas equipadas com controle de luz e temperatura, comedouros e bebedouros e receberam dietas experimentais a vontade. Utilizou-se o método tradicional de coleta total de excreta, com os pintos recebendo a raçãoreferência até a idade de 13 dias, quando se iniciou o experimento. O trabalho foi conduzido no período de oito dias, com temperatura média diária de $33^{\circ} \mathrm{C}$ no interior das gaiolas.

Os alimentos testados substituíram 40\% (base da matéria natural) de uma dieta-referência (Tab. 1) formulada à base de milho moído, farelo de soja, óleo de soja e mistura vitamínico-mineral, constituindo assim sete dietas experimentais. A dieta-referência constituiu um tratamento e foi calculada segundo recomendações de Rostagno et al. (1985). As rações testadas foram consumidas à vontade durante oito dias, em regime de luz de 23 horas. 
As aves foram pesadas no início e final do experimento e passaram por um período de adaptação à dieta de três dias imediatamente anterior ao período de coleta experimental. As excretas foram coletadas diariamente em intervalos de 12 horas durante cinco dias, pesadas, guardadas em sacos plásticos e armazenadas à temperatura de $-20^{\circ} \mathrm{C}$ em congelador até o final do experimento. Para a coleta total de excretas foram utilizadas bandejas colocadas em cada compartimento, cobertas com lona plástica a fim de evitar perdas. Ao final do experimento foram descongeladas, reunidas por repetição, pesadas e homogeneizadas. Retirou-se uma alíquota de $500 \mathrm{~g}$ para secagem em estufa de ventilação forçada à temperatura de $65^{\circ} \mathrm{C}$ durante 72 horas e reservada para análises posteriores. Registraram-se as quantidades da ração-teste ingeridas por unidade experimental, tomando-se o cuidado de separar e pesar as sobras retidas nas bandejas de excreta.

Tabela 1. Composição da ração-referência ${ }^{1}$, em percentagem da matéria seca

\begin{tabular}{lcc}
\hline Ingrediente & $\mathrm{kg}$ \\
\hline Milho moído & 52,000 \\
Farelo de soja & 41,867 \\
Óleo de soja & 2,533 \\
Suplemento vitamínico e mineral ${ }^{2}$ & 0,600 \\
Fosfato bicálcio & 2,400 \\
DL Metionina & 0,200 \\
Sal & \multicolumn{2}{c}{0,400} \\
Composição calculada ${ }^{1}$ & Matéria & Matéria \\
& natural & seca \\
Proteína bruta (\%) & 23,00 & 25,84 \\
Energia metabolizável (kcal/kg) & 2930 & 3300 \\
Cálcio total (\%) & 1,00 & 1,12 \\
Fósforo disponível (\%) & 0,57 & 0,64 \\
Fósforo total (\%) & 0,83 & 0,92 \\
\hline${ }^{1}$ Valores calculados segundo Rostagno et al. (1985) \\
${ }^{2}$ Produto comercial & \multicolumn{2}{c}{}
\end{tabular}

As dietas e o delineamento experimental do método 2 foram as mesmas do método 1, exceto que a unidade experimental foi composta por cinco aves, com 39 dias de idade, alojadas em uma mesma gaiola, constituindo uma repetição. O período experimental foi de oito dias. Foram utilizadas 180 aves das quais 160 foram distribuídas aleatoriamente entre os oito tratamentos com quatro repetições cada e as 20 restantes constituíram o tratamento 9, com a finalidade igual à do método 1.

No método 3 foram utilizados 72 aves oriundas dos ensaios anteriores em delineamento experimental inteiramente ao acaso. Sessenta e quatro aves foram distribuídas em oito tratamentos e quatro repetições, com duas aves por unidade experimental, utilizando-se o método da alimentação forçada descrito por Sibbald (1976). Igual aos métodos anteriores, o tratamento 9 (duas aves por repetição $\times$ quatro repetições = oito aves) foi utilizado para estimar as perdas endógenas e metabólicas.

As aves apresentaram média de peso de $2 \mathrm{~kg}$ e oito semanas de idade. O período experimental foi de quatro dias, obedecendo ao seguinte cronograma: todas as aves foram submetidas ao jejum de 30 horas para esvaziamento completo do trato gastrintestinal e após o jejum foram forçadas a ingerir 25 gramas dos alimentos-teste de uma única vez. Diferente dos métodos anteriores, foram utilizados os alimentos puros, sem fração de substituição. As aves do tratamento 9 foram deixadas em jejum por mais 48 horas para determinação das perdas metabólicas e endógenas. As aves tiveram toda a região da cloaca previamente depenada para evitar aderência da excreta.

A alimentação foi feita por um funil de vidro com $5 \mathrm{~mm}$ de diâmetro interno e $40 \mathrm{~cm}$ de comprimento, introduzido dentro do papo, via esôfago. Os alimentos foram introduzidos com o auxílio de um bastão de mesmo comprimento e 4,5mm de diâmetro. A duração do período da alimentação forçada em cada frango foi, em média, de cinco minutos. 
A coleta de excreta foi total, de 12 em 12 horas, até completar o período exato de 48 horas para todas as aves, inclusive as em jejum. No final do experimento foram agrupadas por repetição e homogeneizadas para futuras análises.

No método 4, com quatro dias de duração, utilizaram-se aves previamente cecectomizadas, segundo Pupa et al. (1998), em delineamento semelhante ao método 3. As aves foram submetidas à cirurgia para retirada dos cecos com procedimentos pré-operatórios de jejum de 24 horas e depenagem da área da cloaca. O pós-operatório foi realizado com injeções intramusculares de penicilina em três dias alternados e retirada dos pontos 12 dias após a cirurgia.

Foram utilizadas 36 aves com 12 semanas de idade e peso médio de 3,5kg. Trinta e duas aves foram distribuídas em oito tratamentos com quatro repetições. As quatro aves restantes foram deixadas em jejum durante todo o período experimental, para obtenção das perdas metabólicas e endógenas. A unidade experimental foi composta de apenas uma ave e as 32 aves foram forçadas a ingerir 25 gramas dos alimentos experimentais puros, semelhante ao método 3.

As análises químicas foram realizadas no laboratório de nutrição animal da Escola de Veterinária da UFMG, adotando-se os seguintes procedimentos para as amostras de alimentos puros, ração-referência e excretas: matéria seca (MS), determinada em estufa de $105^{\circ} \mathrm{C}$ e $55^{\circ} \mathrm{C}$ segundo Cunniff (1995); nitrogênio total ou proteína bruta (PB), determinada pelo método de Kjeldahl segundo Cunniff (1995); energia bruta (EB), utilizando-se calorímetro adiabático PARR.

As análises estatísticas foram feitas no Laboratório de Computação Científica da Universidade Federal de Minas Gerais, em computador IBM 4341. O delineamento experimental foi inteiramente ao acaso, em esquema fatorial de $4 \times 8$ (métodos $\times$ alimentos), perfazendo 32 tratamentos com quatro repetições. As médias foram comparadas pelo teste SNK.

As equações utilizadas no cálculo de energia metabolizável (EMA, EMA $A_{n}, E M V$ e $\operatorname{EMV}_{N}$ ) e para o balanço de nitrogênio (BN) foram as citadas por Albino (1991).

\section{RESULTADOS E DISCUSSÃO}

Os valores de composição química e de energia bruta da ração-referência e dos alimentos encontram-se na Tab. 2. Os alimentos apresentam composições químicas bastante distintas, principalmente quanto aos valores de fibra e amido e, em menor grau, proteína e extrato etéreo. Estes resultados estão de acordo com os de Colnago et al. (1979), Mittelstaedt e Teeter (1993) e McNab (1996), os quais citam que a composição química dos alimentos vegetais é influenciada por uma série de fatores, como por exemplo, variedade, clima, solo, processamentos etc. No caso de subprodutos é influenciada basicamente pelo processamento. Os valores encontrados para os farelos de trigo neste experimento aproximam-se daqueles citados na tabelas nacionais (Rostagno et al., 1985) e estrangeiras (Nutrient..., 1994). Albino (1980) encontrou valores de 18,5\% de PB, 10,2\% de FB e $4649 \mathrm{kcal} / \mathrm{kg}$ de EB na MS de um farelo de trigo para utilização animal. 


\section{Borges et al.}

Tabela 2. Análise química (\%) e valor de energia bruta (kcal/g) segundo o alimento

\begin{tabular}{|c|c|c|c|c|c|c|c|c|c|}
\hline Alimento $^{1}$ & MS & EB & PB & FDN & FDA & FB & $\mathrm{Cz}$ & Amido & $\overline{E E}$ \\
\hline RR & 88,84 & 4521,2 & 25,74 & 15,35 & 0,56 & 3,06 & 6,60 & 47,25 & 3,51 \\
\hline TIM & 86,76 & 5068,3 & 22,10 & 16,43 & 1,73 & 1,84 & 1,68 & 56,24 & 1,90 \\
\hline GT & 89,05 & 4333,6 & 32,02 & 8,39 & ND & ND & 4,95 & 53,43 & 10,4 \\
\hline FTC & 87,34 & 4654,5 & 14,13 & 6,64 & ND & ND & 0,56 & 87,54 & 1,02 \\
\hline FTE & 88,22 & 4508,2 & 18,33 & 4,73 & ND & 0,48 & 1,77 & 82,71 & 3,24 \\
\hline FTH & 89,39 & 4277,4 & 19,63 & 37,03 & 6,82 & 7,71 & 4,97 & 32,47 & 5,12 \\
\hline FTA & 89,50 & 4488,7 & 18,07 & 44,25 & 9,53 & 10,12 & 5,58 & 30,30 & 4,07 \\
\hline FTG & 87,99 & 4681,8 & 18,50 & 41,68 & 7,22 & 8,40 & 5,85 & 32,38 & 4,04 \\
\hline
\end{tabular}

Dados expressos na base da matéria seca.

1: RR= ração-referência, TIM= trigo integral moído, GT= germe de trigo, FTC= farinha de trigo clara, FTE= farinha de trigo escura, $\mathrm{FTH}=$ farelo de trigo para uso humano, FTA= farelo de trigo para uso animal, FTG= farelo de trigo grosso; $\mathrm{MS}=$ matéria seca, EB= energia bruta, $\mathrm{PB}=$ proteína bruta, $\mathrm{FDN}=$ fibra detergente neutro, $\mathrm{FDA}=$ fibra detergente ácido, $\mathrm{FB}=$ fibra bruta, $\mathrm{Cz}=\mathrm{cinzas}, \mathrm{EE}=$ extrato etéreo, $\mathrm{ND}=$ não detectado pelos limites da técnica.

Os valores de energia metabolizável aparente dos alimentos encontram-se na Tab. 3. Os ensaios realizados pelo método tradicional (Trat. 1 e 2) apresentaram maiores valores de EMA para todos os alimentos, quando comparados aos de alimentação forçada (Trat. 3 e 4). Entre os métodos 1 e 2 ocorreram diferenças $(\mathrm{P}<0,05)$ com o trigo integral, farelo de trigo para uso animal e farelo de trigo grosso. O maior valor de EMA do TIM foi com o método 1. Como os farelos apresentam maior teor de fibra e os resultados mais altos de EMA para FTA e FTG foram obtidos no método 2, isto leva a crer que, possivelmente, as aves mais velhas aproveitaram melhor os alimentos mais fibrosos, concordando com Albino et al. (1981), Charalambous e Daghir (1976) e Härtel (1986), que afirmaram haver relação altamente significativa entre alimentos fibrosos e idade das aves.

Os baixos valores de EMA nos métodos com alimentação forçada foram atribuídos à baixa ingestão de alimentos pelas aves. Nesse caso, as perdas fecal e metabólica afetaram sobremaneira os resultados de EMA, o que não ocorreu na metodologia tradicional, na qual o volume ingerido e excretado é grande. Resultados semelhantes foram obtidos por Albino $(1980,1991)$.

Tabela 3. Valores de energia metabolizável aparente (kcal/kg de MS) segundo o alimento e a metodologia experimental

\begin{tabular}{|c|c|c|c|c|}
\hline \multirow[t]{2}{*}{ Alimento $^{1}$} & \multicolumn{2}{|c|}{$\begin{array}{c}\text { Metodologia } \\
\text { tradicional }\end{array}$} & \multicolumn{2}{|c|}{$\begin{array}{c}\text { Alimentação } \\
\text { forçada }\end{array}$} \\
\hline & Trat. 1 & Trat. 2 & Trat. 3 & Trat. 4 \\
\hline$\overline{R R}$ & 3351a & 3384a & $2357 b$ & $2170 \mathrm{c}$ \\
\hline TIM & 4221a & $4114 b$ & 3217 c & 2932d \\
\hline GT & 3006a & 2948a & 2086b & 1991b \\
\hline FTC & 4046a & 4025a & $3156 b$ & $2783 c$ \\
\hline FTE & $3907 a$ & 3934a & $2830 \mathrm{~b}$ & 2527c \\
\hline FTH & $1686 a$ & 1706a & $949 b$ & $1013 b$ \\
\hline FTA & 1931b & 2097a & $1108 d$ & 1250c \\
\hline FTG & $2028 b$ & 2223a & $1336 c$ & $1170 \mathrm{~d}$ \\
\hline $\mathrm{R}^{2}$ & 0,99 & 0,99 & 0,99 & 0,99 \\
\hline CV (\%) & 3,00 & 2,59 & 3,03 & 3,10 \\
\hline
\end{tabular}

Valores na mesma linha seguidos de letras distintas diferem entre si pelo teste $\mathrm{SNK}(\mathrm{P}<0,05)$.

1: RR= ração-referência, TIM= trigo integral moído, GT= germe de trigo, FTC= farinha de trigo clara, FTE= farinha de trigo escura, $\mathrm{FTH}=$ farelo de trigo para uso humano, FTA= farelo de trigo para uso animal, FTG= farelo de trigo grosso.

Entre os métodos 3 e 4 ocorreram diferenças. As aves do método 4 apresentaram menores valores de EMA para os alimentos RR, TIM, FTC, FTE e FTG e maior valor para FTA. Não há explicação plausível para estes resultados, uma vez que a EMA de ambos é bastante afetada pelas perdas metabólica e endógena, as quais podem apresentar ampla variação individual (Flores, Castanon, 1991). Além disso, a 
diferença de peso das aves nos métodos 3 e 4 pode ter sido um fator de confudimento. A correção a partir da excreta de aves em jejum (EMV) reflete melhor o efeito da cecectomia sobre o aproveitamento energético das aves. De qualquer forma, a metodologia de Sibbald (1976) subestimou os valores de EMA, conforme dados obtidos neste e em outros trabalhos como, por exemplo, em Albino (1991).

Quanto à EMV, observaram-se diferenças $(\mathrm{P}<0,05)$ entre os tratamentos (Tab. 4), porém menos acentuadas que as observadas para a EMA. Os ensaioss com a metodologia tradicional não apresentaram resultados superiores em relação aos de metodologia de alimentação forçada, pelo contrário, eles foram iguais ou mesmo inferiores. Albino (1991) encontrou resultados semelhantes e ponderou que isso se deveu à correção feita pela excreta das aves em jejum, que anulou o efeito do baixo nível de consumo.

Tabela 4. Valores de energia metabolizável verdadeira ( $\mathrm{kcal} / \mathrm{kg}$ de MS) segundo o alimento e a metodologia experimental

\begin{tabular}{|c|c|c|c|c|}
\hline \multirow[t]{2}{*}{ Alimento $^{1}$} & \multicolumn{2}{|c|}{$\begin{array}{l}\text { Metodologia } \\
\text { tradicional }\end{array}$} & \multicolumn{2}{|c|}{$\begin{array}{l}\text { Alimentação } \\
\text { forçada }\end{array}$} \\
\hline & Trat. 1 & Trat. 2 & Trat. 3 & Trat. 4 \\
\hline $\mathrm{RR}$ & $3417 a$ & $3416 a$ & $3341 a$ & $3388 a$ \\
\hline TIM & 4273a & 4137a & $4224 a$ & 4179a \\
\hline GT & 3094ab & 2971b & 3068ab & 3206а \\
\hline FTC & 4113a & $4066 a$ & $4156 a$ & $4022 a$ \\
\hline FTE & 3971a & $3962 a$ & $3820 a b$ & $3753 b$ \\
\hline FTH & $1767 c$ & $1737 \mathrm{c}$ & $1927 b$ & 2223a \\
\hline FTA & 2002b & $2120 b$ & 2084b & 2459a \\
\hline FTG & $2107 c$ & $2250 b$ & 2329ab & $2400 a$ \\
\hline $\mathrm{R}^{2}$ & 0,993 & 0,994 & 0,996 & 0,995 \\
\hline CV (\%) & 2,87 & 2,56 & 2,07 & 1,91 \\
\hline
\end{tabular}

Valores em uma mesma linha seguidos de letras distintas diferem entre si pelo teste $\mathrm{SNK}(\mathrm{P}<0,05)$.

1: $\mathrm{RR}=$ ração-referência, TIM= trigo integral moído, GT= germe de trigo, FTC= farinha de trigo clara, FTE= farinha de trigo escura, $\mathrm{FTH}=$ farelo de trigo para uso humano, FTA= farelo de trigo para uso animal, FTG= farelo de trigo grosso.

Na metodologia tradicional, os valores de EMV de todos os alimentos foram semelhantes ( $\mathrm{P}>0,05)$, com exceção ao FTG que apresentou valor mais alto no método 2 . Na alimentação forçada ocorreram diferenças significativas para FTE, FTA e FTH, isto é, maiores valores de EMV no método 4, com aves cecectomizadas. Estes resultados são diferentes dos de Hayes et al. (1990), os quais sugerem que aves cecectomizadas apresentariam menor valor de energia metabolizável, pois as bactérias do ceco fermentariam a fibra e outros resíduos sobrepassantes ao intestino delgado, transformando-os em AGV, e a excreta apresentaria menor valor energético, o que determinaria maior teor de energia do alimento.

Na Tab. 5 encontram-se os valores da $\mathrm{EMA}_{n}$ dos alimentos. Semelhante à EMA, os valores pelo método tradicional foram superiores $(\mathrm{P}<0,05)$ aos dos métodos de alimentação forçada, o que era esperado, uma vez que as correções pelo nitrogênio não eliminam os efeitos da baixa ingestão de alimentos. Comparando com os valores de EMA, observa-se que os de $\mathrm{EMA}_{\mathrm{n}}$ em todos os métodos apresentaram tendência de redução, não avaliada estatisticamente, o que pode significar que as aves apresentaram balanço positivo de N, fato já verificado por Sibbald e Wolynetz (1985). 


\section{Borges et al.}

Tabela 5. Valores de energia metabolizável aparente corrigida (kcal/kg de MS) segundo o alimento e a metodologia experimental

\begin{tabular}{|c|c|c|c|c|}
\hline \multirow[t]{2}{*}{ Alimento $^{1}$} & \multicolumn{2}{|c|}{$\begin{array}{l}\text { Metodologia } \\
\text { tradicional }\end{array}$} & \multicolumn{2}{|c|}{$\begin{array}{l}\text { Alimentação } \\
\text { forçada }\end{array}$} \\
\hline & Trat. 1 & Trat. 2 & Trat. 3 & Trat. 4 \\
\hline$\overline{R R}$ & $3121 a$ & $3200 a$ & $2084 b$ & $1785 c$ \\
\hline TIM & 4059a & 4001a & $3003 b$ & $2728 c$ \\
\hline GT & $2758 a$ & $2785 a$ & $1846 b$ & $1620 \mathrm{c}$ \\
\hline FTC & 3898a & 3881a & 2838b & $2683 c$ \\
\hline FTE & $3706 a$ & $3754 a$ & $2576 b$ & $2329 c$ \\
\hline FTH & $1557 a$ & $1614 a$ & 860b & $710 c$ \\
\hline FTA & $1789 b$ & $1938 a$ & $982 \mathrm{c}$ & $718 d$ \\
\hline FTG & 1911b & 2105a & $1148 c$ & 863d \\
\hline $\mathrm{R}^{2}$ & 0,99 & 0,99 & 0,99 & 0,98 \\
\hline CV (\%) & 2,99 & 2,56 & 3,56 & 8,00 \\
\hline
\end{tabular}

Valores na mesma linha seguidos de letras distintas diferem entre si pelo teste SNK $(\mathrm{P}<0,05)$.

1: $\mathrm{RR}=$ ração-referência, TIM= trigo integral moído, GT= germe de trigo, FTC= farinha de trigo clara, FTE= farinha de trigo escura, $\mathrm{FTH}=$ farelo de trigo para uso humano, FTA= farelo de trigo para uso animal, FTG= farelo de trigo grosso.

Albino (1991) obteve resultados diferentes, com maiores valores de $\mathrm{EMA}_{\mathrm{n}}$ nos métodos com alimentação forçada, isto é, as aves apresentaram valores negativos de N. O autor atribui esse fato ao baixo consumo de alimento e ao período de jejum ao qual as aves foram submetidas. Nesta pesquisa seria de se esperar que as aves dos métodos de alimentação forçada apresentassem valores negativos de BN, o que não ocorreu. O fato é que Albino (1991) utilizou aves adultas com peso médio de 5kg, diferente do presente trabalho, com aves ainda em crescimento, com retenção de proteína, o que pode explicar a menor excreção absoluta de N. Nos tratamentos com o método tradicional os valores de EMA $\mathrm{n}_{\mathrm{n}}$ foram similares, com exceção ao FTA e FTG que apresentaram valores maiores no tratamento 2, do mesmo modo que para EMA, reforçando a idéia de que aves com idade mais avançada aproveitariam melhor alimentos mais fibrosos, semelhante ao encontrado por Shires et al. (1980).

$\mathrm{Na}$ alimentação forçada pode-se observar que as aves não cecectomizadas apresentaram maiores valores de $\mathrm{EMA}_{\mathrm{n}}$ do que as cecectomizadas. Como as aves dos dois tratamentos receberam a mesma quantidade de alimento, pode-se pressupor que esse efeito se deve à diferença na quantidade de perdas metabólica e endógena, naturalmente maiores nas aves do método 4, que eram mais pesadas do que as do método 3. Assim, os valores de EMAn, coerentemente, são menores com a utilização do método 4, independente do fato das aves serem cecectomizadas ou não. Hayes et al. (1990), ao avaliarem alimentos como farelo de soja, torta de girassol, milho e sorgo, encontraram efeito significativo da cecectomia sobre a $\mathrm{EMV}_{\mathrm{n}}$ da torta de girassol e do milho, com menores valores em aves cecectomizadas quando comparadas às não cecectomizadas.

Entre os valores de $\mathrm{EMV}_{\mathrm{n}}$ dos alimentos ocorreram algumas diferenças, embora bem menores do que as encontradas

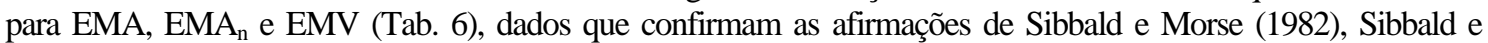
Wolynetz (1985) e Sibbald e Wolynetz (1988), os quais sugeriram ser essa a estimativa mais precisa para a energia metabolizável. Quando se comparam todos os tratamentos, observa-se que os valores de $\mathrm{EMV}_{\mathrm{n}}$ dos alimentos foram semelhantes, com exceção aos valores da RR, TIM e FTH. A RR apresentou menores valores no tratamento com aves cecectomizadas. 
Tabela 6. Valores de energia metabolizável verdadeira corrigida (kcal/kg de MS) segundo o alimento e a metodologia experimental

\begin{tabular}{|c|c|c|c|c|}
\hline \multirow[t]{2}{*}{ Alimento $^{1}$} & \multicolumn{2}{|c|}{$\begin{array}{c}\text { Metodologia } \\
\text { tradicional }\end{array}$} & \multicolumn{2}{|c|}{$\begin{array}{c}\text { Alimentação } \\
\text { forçada }\end{array}$} \\
\hline & Trat. 1 & Trat. 2 & Trat. 3 & Trat. 4 \\
\hline RR & $3187 a b$ & $3231 a$ & 3067bc & $3004 c$ \\
\hline TIM & 4111a & 4024ab & 4010ab & $3976 b$ \\
\hline GT & 2846a & 2809a & 2828a & $2835 a$ \\
\hline FTC & 3966a & 3922a & 3839a & 3923a \\
\hline FTE & 3770a & 3782a & 3566a & $3556 a$ \\
\hline FTH & 1639b & $1646 b$ & 1838a & 1921a \\
\hline FTA & 1860a & $2006 a$ & 1958a & $1927 a$ \\
\hline FTG & 1990a & $2132 a$ & $2141 a$ & 2093a \\
\hline $\mathrm{R}^{2}$ & 0,99 & 0,99 & 0,99 & 0,98 \\
\hline CV (\%) & 2,87 & 2,52 & 2,35 & 4,63 \\
\hline
\end{tabular}

Valores na mesma linha seguidos de letras distintas diferem entre si pelo teste $\mathrm{SNK}(\mathrm{P}<0,05)$.

1: RR= ração-referência, TIM= trigo integral moído, GT= germe de trigo, FTC= farinha de trigo clara, FTE= farinha de trigo escura, $\mathrm{FTH}=$ farelo de trigo para uso humano, FTA= farelo de trigo para uso animal, FTG= farelo de trigo grosso.

O TIM apresentou menor e maior valores nos tratamentos 4 e 1, respectivamente, e valores intermediários nos tratamentos 2 e 3 . Todos os alimentos dos tratamentos pela metodologia tradicional foram semelhantes entre si, embora os farelos apresentassem tendência a menores resultados no tratamento 1 . O mesmo resultado ocorreu com a metodologia de alimentação forçada. Exceção ao FTH, cujo valor foi maior, os valores de $\mathrm{EMV}_{\mathrm{n}}$ no tratamento 3 foram semelhantes ao do tratamento 1 .

Teoricamente todos os tratamentos deveriam apresentar resultados iguais, à semelhança do que ocorreu com os tratamentos 1 e 2, mas apesar de todos os tratamentos apresentaram valores de $\mathrm{R}^{2}$ semelhantes, o maior coeficiente de variação ocorreu nos tratamentos com aves cecectomizadas. Esse coeficiente de variação foi atribuído à quantidade de $\mathrm{N}$ excretada (Tab. 7) que afetou o balanço de nitrogênio (Tab. 8).

A quantidade de $\mathrm{N}$ excretado apresentou maior oscilação individual nos tratamentos com a metodologia de Sibbald (Tab. 7). Essas diferenças individuais e o alto coeficiente de variação podem ter causado erros que refletiram nos valores de $\mathrm{N}$ dos tratamentos de alimentação forçada. Mais ainda, diferenças no peso e no consumo podem ter influenciado a excreção de N. A variabilidade dos dados obtidos em aves em jejum é uma das críticas feitas por Dale e Fuller (1984) à metodologia de Sibbald, que se confirma no presente trabalho. 


\section{Borges et al.}

Tabela 7. Valores de nitrogênio ${ }^{1}$ do alimento e da excreta (\% MS), segundo o alimento e a metodologia experimental

\begin{tabular}{lccccc}
\hline \multirow{2}{*}{ Alimento $^{2} \begin{array}{c}\text { N no } \\
\text { alimento }\end{array}$} & \multicolumn{4}{c}{ N na excreta } \\
\cline { 3 - 6 } & Trat. 1 & Trat. 2 & Trat. 3 & Trat. 4 \\
\hline RR & 4,12 & $5,24 \mathrm{~d}$ & $6,48 \mathrm{c}$ & $11,37 \mathrm{~b}$ & $13,01 \mathrm{a}$ \\
TIM & 2,51 & $4,91 \mathrm{~d}$ & $6,07 \mathrm{c}$ & $12,30 \mathrm{~b}$ & $15,69 \mathrm{a}$ \\
GT & 5,12 & $6,08 \mathrm{~b}$ & $7,49 \mathrm{~b}$ & $13,37 \mathrm{a}$ & $14,43 \mathrm{a}$ \\
FTC & 2,26 & $5,29 \mathrm{c}$ & $6,10 \mathrm{c}$ & $11,45 \mathrm{~b}$ & $18,26 \mathrm{a}$ \\
FTE & 2,93 & $5,24 \mathrm{c}$ & $6,43 \mathrm{c}$ & $12,73 \mathrm{~b}$ & $15,99 \mathrm{a}$ \\
FTH & 3,14 & $3,88 \mathrm{~b}$ & $4,68 \mathrm{~b}$ & $9,28 \mathrm{a}$ & $9,38 \mathrm{a}$ \\
FTA & 2,89 & $3,41 \mathrm{c}$ & $4,41 \mathrm{bc}$ & $8,64 \mathrm{a}$ & $5,75 \mathrm{~b}$ \\
FTG & 2,96 & $3,84 \mathrm{~b}$ & $4,36 \mathrm{~b}$ & $8,06 \mathrm{a}$ & $9,34 \mathrm{a}$ \\
R & & 0,99 & 0,94 & 0,82 & 0,83 \\
CV & - & 2,05 & 5,47 & 9,13 & 16,18
\end{tabular}

Valores na mesma linha seguidos de letras distintas diferem entre si pelo teste $\mathrm{SNK}(\mathrm{P}<0,05)$.

1: expressos em g de N/g de MS de excreta. 2: RR= ração-referência, TIM= trigo integral moído, GT= germe de trigo, FTC= farinha de trigo clara, FTE= farinha de trigo escura, FTH= farelo de trigo para uso humano, FTA= farelo de trigo para uso animal, FTG= farelo de trigo grosso.

Os menores valores de $\mathrm{N}$ na excreta foram observadas nos tratamentos com metodologia tradicional. Entre eles, os frangos (trat. 2) apresentaram maior excreção de $\mathrm{N}$, em peso absoluto, do que os pintos (trat. 1), quanto à RR e TIM (Tab. 7). A razão da porcentagem de $\mathrm{N}$ na excreta ser superior à porcentagem de $\mathrm{N}$ dos alimentos é atribuída à alta excreção de ácido úrico na urina junto as fezes. Como a quantidade de excreta dos tratamentos com base na metodologia tradicional é maior, a porcentagem de $\mathrm{N}$ na excreta é mais diluída. O contrário ocorre nos tratamentos de alimentação forçada. É interessante notar que em alimentos mais fibrosos, cuja quantidade de excreta é maior, a porcentagem de $\mathrm{N}$ é menor devido ao efeito diluente da fibra.

Tabela 8. Valores do balanço de nitrogênio, segundo o alimento e a metodologia experimental

\begin{tabular}{|c|c|c|c|c|}
\hline \multirow[t]{2}{*}{ Alimento $^{1}$} & \multicolumn{2}{|c|}{$\begin{array}{c}\text { Metodologia } \\
\text { tradicional }\end{array}$} & \multicolumn{2}{|c|}{$\begin{array}{l}\text { Alimentação } \\
\text { forçada }\end{array}$} \\
\hline & Trat. 1 & Trat. 2 & Trat. 3 & Trat. 4 \\
\hline$\overline{R R}$ & $2,02 b$ & $3,16 a$ & $1,48 c$ & $1,04 d$ \\
\hline TIM & $1,94 b$ & $2,99 a$ & $1,13 \mathrm{c}$ & $0,54 d$ \\
\hline GT & $1,84 b$ & 3,36a & $1,30 \mathrm{c}$ & $1,01 \mathrm{c}$ \\
\hline FTC & $1,72 b$ & 2,58a & $1,69 b$ & $0,27 \mathrm{c}$ \\
\hline FTE & $1,94 b$ & $3,27 a$ & $1,36 \mathrm{c}$ & $0,54 \mathrm{~d}$ \\
\hline FTH & $1,52 b$ & 2,51a & $0,48 \mathrm{~d}$ & $0,96 c$ \\
\hline FTA & $1,66 \mathrm{~b}$ & 3,05a & $0,68 c$ & $1,58 \mathrm{~b}$ \\
\hline FTG & $1,50 \mathrm{~b}$ & $2,88 a$ & $1,00 \mathrm{c}$ & $0,90 \mathrm{c}$ \\
\hline Média & 1,77 & 2,98 & 1,31 & 0,856 \\
\hline $\mathrm{R}^{2}$ & 0,92 & 0,77 & 0,66 & 0,61 \\
\hline CV (\%) & 3,63 & 6,02 & 27,56 & 40,40 \\
\hline
\end{tabular}

Valores na mesma linha seguidos de letras distintas diferem entre si pelo teste SNK $(\mathrm{P}<0,05)$.

1: RR= ração-referência, TIM= trigo integral moído, GT= germe de trigo, FTC= farinha de trigo clara, FTE= farinha de trigo escura, $\mathrm{FTH}$ = farelo de trigo para uso humano, FTA= farelo de trigo para uso animal, FTG= farelo de trigo grosso.

Na Tab. 9 encontram-se valores da relação entre energia metabolizável verdadeira e aparente. Na metodologia tradicional os valores são próximos de um, demonstrando estreita relação entre as duas medidas, enquanto que na metodologia de Sibbald os valores são maiores, principalmente naqueles alimentos cuja energia metabolizável foi mais baixa, como os farelos, por exemplo. Estes resultados são esperados uma vez que a influência das perdas endógena e metabólica se evidencia no baixo consumo, 
como o que ocorre na metodologia de Sibbald, tornando-se muito elevada a diferença entres as medidas de EMA e EMV. Os coeficientes de variação foram baixos, com o maior valor nas aves cecectomizadas.

Tabela 9. Relação entre energia metabolizável verdadeira (EMV) e energia metabolizável aparente (EMA) segundo o alimento e a metodologia experimental

\begin{tabular}{|c|c|c|c|c|}
\hline \multirow{3}{*}{ Alimento $^{1}$} & \multicolumn{4}{|c|}{ Relação EMV/EMA } \\
\hline & \multicolumn{2}{|c|}{$\begin{array}{l}\text { Metodologia } \\
\text { tradicional }\end{array}$} & \multicolumn{2}{|c|}{$\begin{array}{l}\text { Alimentação } \\
\text { forçada }\end{array}$} \\
\hline & Trat. 1 & Trat. 2 & Trat. 3 & Trat. 4 \\
\hline $\mathrm{RR}$ & $1,020 \mathrm{c}$ & $1,0092 \mathrm{c}$ & $1,418 \mathrm{~b}$ & $1,561 a$ \\
\hline TIM & $1,012 \mathrm{c}$ & $1,0056 \mathrm{c}$ & $1,313 b$ & $1,426 \mathrm{a}$ \\
\hline GT & $1,029 \mathrm{c}$ & $1,0079 d$ & $1,470 \mathrm{~b}$ & $1,610 \mathrm{a}$ \\
\hline FTC & $1,016 \mathrm{c}$ & $1,0100 d$ & $1,317 b$ & $1,445 a$ \\
\hline FTE & $1,016 \mathrm{c}$ & $1,0071 \mathrm{c}$ & $1,350 \mathrm{~b}$ & $1,486 a$ \\
\hline FTH & $1,048 \mathrm{c}$ & $1,0186 \mathrm{c}$ & $2,033 b$ & $2,199 a$ \\
\hline FTA & $1,036 \mathrm{c}$ & $1,0104 \mathrm{c}$ & $1,883 b$ & $1,969 a$ \\
\hline FTG & $1,039 c$ & $1,0119 \mathrm{c}$ & $1,746 b$ & 1,993a \\
\hline CV (\%) & 0,23 & 0,057 & 2,26 & 3,98 \\
\hline $\mathrm{R}^{2}$ & 0,97 & 0,98 & 0,99 & 0,96 \\
\hline
\end{tabular}

Valores na mesma linha seguidos de letras distintas diferem entre si pelo teste $\mathrm{SNK}(\mathrm{P}<0,05)$.

1: RR= ração-referência, TIM= trigo integral moído, GT =germe de trigo, FTC= farinha de trigo clara, FTE= farinha de trigo escura, FTH= farelo de trigo para uso humano, FTA= farelo de trigo para uso animal, FTG= farelo de trigo grosso.

Na Tab. 10 encontram-se valores da relação entre $\mathrm{EMV}_{\text {trat.3}} / \mathrm{EMA}_{\text {trat.1 }}$ e $\mathrm{EMV}_{\text {trat.3 }} / \mathrm{EMA}_{\text {trat.2 }}(1,03$ e 1,02, respectivamente) e entre $\mathrm{EMV}_{\text {trat.3}} / \mathrm{EMV}_{\text {trat.1 }}$ e $\mathrm{EMV}_{\text {trat.3 }} / \mathrm{EMV}_{\text {trat.1 }}$ (mesmo fator, 1,01). Entretanto, dependendo da natureza do alimento, esse fator pode variar bastante; por exemplo, o fator para a relação EMV $_{\text {trat.3 }} /$ EMA $_{\text {trat.1 }}$ oscilou de 0,98 (FTE) a 1,15 (FTG).

Os valores da relação entre $\mathrm{EMV}_{\mathrm{n}}$ e $\mathrm{EMA}_{\mathrm{n}}$ são apresentados na Tab. 11. Nos tratamentos 1 e 2 as relações foram próximas de um, entretanto, nos tratamentos 3 e 4 essas relações variaram largamente de acordo com o alimento. Os coeficientes de variação na metodologia tradicional foram muito baixos, e nos de alimentação forçada, um pouco mais altos.

Sibbald (1977), apud Albino et al. (1994), propõe a utilização de fatores de correção para estimar a EMV dos alimentos a partir da EMA, ou vice versa, assim, o ensaio de EMV, por ser relativamente barato e rápido poderia ser utilizado para avaliar a disponibilidade energética de rações para aves.

Tabela 10. Relação entre energia metabolizável verdadeira (EMV) e energia metabolizável aparente (EMA) segundo o alimento

\begin{tabular}{lcccc}
\hline Alimento & $\mathrm{EMV}_{\text {trat.3 }} / \mathrm{EMA}_{\text {trat.1 }}$ & $\mathrm{EMV}_{\text {Trat.3 }} / \mathrm{EMA}_{\text {trat.2 }}$ & $\mathrm{EMV}_{\text {Trat.3 }} / \mathrm{EMV}_{\text {trat.1 }}$ & $\mathrm{EMV}_{\text {Trat.3 }} / \mathrm{EMV}_{\text {trat.2 }}$ \\
\hline RR & 1,00 & 0,99 & 0,98 & 0,98 \\
TIM & 1,00 & 1,03 & 0,99 & 1,02 \\
GT & 1,02 & 1,04 & 0,99 & 1,03 \\
FTC & 1,03 & 1,03 & 1,01 & 1,02 \\
FTE & 0,98 & 0,97 & 0,96 & 0,96 \\
FTH & 1,14 & 1,13 & 1,09 & 1,11 \\
FTA & 1,08 & 0,99 & 1,04 & 0,98 \\
FTG & 1,15 & 1,05 & 1,11 & 1,04 \\
R & 0,07 & 0,05 & 0,05 & 0,05 \\
CV (\%) & 6,45 & 4,82 & 5,27 & 4,51 \\
\hline
\end{tabular}

1: RR= ração-referência, TIM= trigo integral moído, GT= germe de trigo, FTC= farinha de trigo clara, FTE= farinha de trigo escura, $\mathrm{FTH}=$ farelo de trigo para uso humano, FTA= farelo de trigo para uso animal, FTG= farelo de trigo grosso. 


\section{Borges et al.}

Tabela 11. Relação entre energia metabolizável verdadeira corrigida $\left(\mathrm{EMV}_{\mathrm{n}}\right)$ e energia metabolizável aparente corrigida $\left(\mathrm{EMA}_{\mathrm{n}}\right)$ segundo o alimento e a metodologia experimental

\begin{tabular}{|c|c|c|c|c|}
\hline \multirow{3}{*}{ Alimento $^{1}$} & \multicolumn{4}{|c|}{ Relação $\mathrm{EMV}_{\mathrm{n}} / \mathrm{EMA}_{\mathrm{n}}$} \\
\hline & \multicolumn{2}{|c|}{$\begin{array}{l}\text { Metodologia } \\
\text { tradicional }\end{array}$} & \multicolumn{2}{|c|}{$\begin{array}{c}\text { Alimentação } \\
\text { forçada }\end{array}$} \\
\hline & Trat.1 & Trat.2 & Trat.3 & Trat.4 \\
\hline RR & $1,021 \mathrm{c}$ & $1,010 \mathrm{c}$ & $1,473 b$ & 1,683a \\
\hline TIM & $1,013 \mathrm{c}$ & $1,006 \mathrm{~d}$ & $1,335 b$ & $1,457 a$ \\
\hline GT & $1,032 \mathrm{c}$ & $1,008 \mathrm{c}$ & $1,532 b$ & $1,753 a$ \\
\hline FTC & $1,017 \mathrm{c}$ & $1,010 \mathrm{c}$ & $1,353 b$ & $1,462 \mathrm{a}$ \\
\hline FTE & $1,017 \mathrm{c}$ & $1,008 \mathrm{c}$ & $1,385 b$ & $1,529 a$ \\
\hline FTH & $1,052 \mathrm{c}$ & $1,020 \mathrm{c}$ & $2,140 \mathrm{~b}$ & 2,723a \\
\hline FTA & $1,039 \mathrm{c}$ & $1,011 \mathrm{c}$ & $1,995 b$ & $2,729 a$ \\
\hline FTG & $1,041 \mathrm{c}$ & $1,013 c$ & $1,967 b$ & $2,368 a$ \\
\hline CV (\%) & 0,26 & 0,060 & 2,18 & 10,44 \\
\hline $\mathrm{R}^{2}$ & 0,97 & 0,98 & 0,99 & 0,89 \\
\hline
\end{tabular}

Valores na mesma linha seguidos de letras distintas diferem entre si pelo teste $\mathrm{SNK}(\mathrm{P}<0,05)$.

1: RR = ração-referência, TIM = trigo integral moído, GT =germe de trigo, FTC =farinha de trigo clara, FTE = farinha de trigo escura, FTH = farelo de trigo para uso humano, FTA = farelo de trigo para uso animal, FTG = farelo de trigo grosso.

Quando se aplicou a correção pelo nitrogênio (Tab. 12) encontraram-se valores médios de 1,02 para

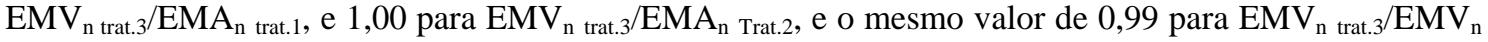
trat.1 e 2, mas com grande variação entre os alimentos, semelhante ao ocorrido sem a correção de $\mathrm{N}$ (Tab. 10). Desse modo, a utilização de fatores como proposto por Sibbald (1977), apud Albino (1991), parece não ser um bom fator de predição para a EMV e $E M V_{n}$ dos alimentos, uma vez que eles podem subestimar ou superestimar a energia, dependendo da natureza do alimento. Os coeficientes de variação das relações $\mathrm{EMV}_{\text {trat.3}} / \mathrm{EMA}_{\text {trat.1 }}$ e EMV trat.3/ $\mathrm{EMV}_{\text {trat.1 }}$ apresentaram tendência (não avaliada estaticamente) de serem maiores do que os coeficientes das relações $E_{M V} V_{\text {trat.3 }} / \mathrm{EMA}_{\text {trat.2 }}$ e $\mathrm{EMV}_{\text {trat.3 }} / \mathrm{EMV}_{\text {trat.2. }}$. Possivelmente isso pode ser atribuído ao efeito de idade, uma vez que a relação entre trat.3/trat.1 associa pintos a frangos, enquanto que a relação trat.3/trat.2 associa aves com idades próximas.

Tabela 12. Relação entre energia metabolizável verdadeira corrigida pelo nitrogênio (EMVn) e energia metabolizável aparente corrigida pelo nitrogênio (EMAn) segundo o alimento

\begin{tabular}{lcccc}
\hline Alimento & $\mathrm{EMV}_{\mathrm{n} \text { trat.3 }} / \mathrm{EMA}_{\mathrm{n} \text { trat.1 }}$ & $\mathrm{EMV}_{\mathrm{n} \text { trat.3 }} / \mathrm{EMA}_{\mathrm{n} \text { trat.2 }}$ & $\mathrm{EMV}_{\mathrm{n}}$ trat.3/ $\mathrm{EMV}_{\mathrm{n} \text { trat.1 }}$ & $\mathrm{EMV}_{\mathrm{n} \text { trat.3 }} / \mathrm{EMV}_{\mathrm{n} \text { trat.2 }}$ \\
\hline RR & 0,98 & 0,96 & 0,96 & 0,96 \\
TIM & 0,99 & 1,00 & 0,98 & 0,98 \\
$\mathrm{GT}$ & 1,03 & 1,02 & 0,99 & 0,99 \\
FTC & 0,98 & 0,99 & 0,97 & 0,97 \\
FTE & 0,96 & 0,95 & 0,95 & 0,95 \\
FTH & 1,18 & 1,14 & 1,12 & 1,12 \\
FTA & 1,09 & 1,01 & 1,05 & 1,05 \\
FTG & 1,12 & 1,02 & 1,08 & 1,08 \\
$\mathrm{R}^{2}$ & 0,08 & 0,06 & 0,06 & 0,06 \\
$\mathrm{CV}(\%)$ & 7,81 & 5,79 & 6,38 & 6,38 \\
\hline
\end{tabular}

1: RR= ração-referência, TIM= trigo integral moído, GT= germe de trigo, $\mathrm{FTC}=$ farinha de trigo clara, $\mathrm{FTE}=$ farinha de trigo escura, $\mathrm{FTH}=$ farelo de trigo para uso humano, FTA= farelo de trigo para uso animal, FTG= farelo de trigo grosso.

Os valores de MS, N e energia expressos por ave, por 100 gramas de peso vivo e por peso metabólico das aves em jejum são apresentados na Tab. 13. Os valores de MS nas excretas das aves em jejum, quando expressos em valores absolutos (g/ave/dia), apresentam-se menores nos tratamentos com metodologia tradicional e maiores nos tratamentos de alimentação forçada, e se relacionam diretamente com o peso corporal. Albino (1991) encontrou resultados semelhantes e afirmou que as perdas relacionam-se diretamente com o peso e a idade das aves. Entretanto, quando as perdas foram calculadas com base em g/100g de ave e no peso metabólico, observa-se que os pintos (tratamento 1) apresentaram maiores 
valores, explicado pelo fato de o metabolismo protéico nas aves jovens ser maior do que nas aves adultas, as quais apresentam maior anabolismo e catabolismo.

Tabela 13. Matéria seca (MS), nitrogênio (N) e energia bruta (EB) da excreta de aves em jejum, expressas por ave, por 100 gramas de peso vivo (PV) e por peso metabólico

\begin{tabular}{|c|c|c|c|c|}
\hline \multirow{2}{*}{ Tratamento } & \multirow{2}{*}{ Peso das aves (g) } & \multicolumn{3}{|c|}{ MS excretada em 24h (g) } \\
\hline & & Por ave & Por 100g PV & Por $\mathrm{PV}^{0,75}$ \\
\hline Trat. 1 & 450,750 & $1,39 c$ & $0,31 a$ & $2,51 \mathrm{a}$ \\
\hline Trat. 2 & 1402,500 & $1,24 \mathrm{c}$ & $0,08 \mathrm{c}$ & $0,96 \mathrm{c}$ \\
\hline Trat. 3 & 1968,250 & $3,19 b$ & $0,16 b$ & $1,91 b$ \\
\hline Trat. 4 & 3292,750 & 4,61a & $0,14 b$ & $1,88 \mathrm{~b}$ \\
\hline \multirow[t]{3}{*}{ CV (\%) } & & 12,23 & 15,52 & 13,86 \\
\hline & \% N na MS da excreta & \multicolumn{3}{|c|}{ N excretado em 24h (g) } \\
\hline & & Por ave & Por $100 \mathrm{~g}$ PV & Por PV ${ }^{0,75}$ \\
\hline Trat. 1 & 19,32 & $0,27 c$ & $0,060 \mathrm{a}$ & $0,48 a$ \\
\hline Trat. 2 & 18,54 & $0,23 c$ & $0,016 \mathrm{c}$ & $0,20 b$ \\
\hline Trat. 3 & 20,01 & $0,64 \mathrm{~b}$ & $0,032 b$ & $0,38 a$ \\
\hline Trat. 4 & 22,23 & $1,03 a$ & $0,031 b$ & $0,42 \mathrm{a}$ \\
\hline \multirow[t]{3}{*}{$\mathrm{CV}(\%)$} & 9,42 & 17,64 & 23,11 & 19,66 \\
\hline & & \multicolumn{3}{|c|}{ EB excretada em 24h (kcal) } \\
\hline & & Por ave & Por $100 \mathrm{~g}$ PV & Por $\mathrm{PV}^{0,75}$ \\
\hline Trat. 1 & & $4,75 c$ & $1,05 a$ & $8,62 a$ \\
\hline Trat. 2 & & $4,42 c$ & $0,31 c$ & $3,42 c$ \\
\hline Trat. 3 & & $10,92 b$ & $0,55 b$ & $6,54 b$ \\
\hline Trat. 4 & & $13,52 a$ & $0,41 b$ & $5,53 \mathrm{~b}$ \\
\hline $\mathrm{CV}(\%)$ & & 18,69 & 14,75 & 14,79 \\
\hline
\end{tabular}

Valores na mesma coluna, para uma mesma variável, seguidos de letras distintas diferem entre si, pelo teste SNK $(\mathrm{P}<0,05)$.

As aves cecectomizadas apresentaram maior quantidade de perdas metabólica e endógena de matéria seca, nitrogênio e energia bruta quando comparadas por gramas de excreta/ aves em 24 horas. Hayes et al. (1990) citam que os microorganismos das aves não cecectomizadas em jejum utilizam o material oriundo das perdas fecais (como descamação e muco) para obtenção de energia, apresentando menor excreção final de perdas endógenas e metabólicas. Ao contrário, as aves cecectomizadas não promovem fermentação do material metabólico, apresentando maior excreção de perdas.

Os resultados de $\mathrm{N}$ e de MS apresentaram comportamento semelhante, com valores altos para aves cecectomizadas (tratamento 4) quando expressos em termos absolutos e valores altos para pintos (tratamento 1) quando expressos em g/100g. Quantos aos valores de $\mathrm{N}$ expressos em $\mathrm{P}^{0,75}$, as aves apresentaram valores iguais, com exceção ao tratamento 2, que apresentou valores menores.

Os valores de energia bruta (EB) das aves em jejum têm resultados semelhantes aos encontrados para MS e N. Quando a EB excretada foi expressa em termos absolutos (kcal por ave em 24 horas) as aves cecectomizadas apresentaram maior excreção endógena e metabólica de energia, entretanto quando foram expressas por g/100g de aves os pintos apresentam maiores perdas energéticas.

\section{CONCLUSÕES}

Nas condições em que foram realizados estes tratamentos pode-se concluir que: 1- a energia metabolizável aparente reflete bem a energia disponível dos alimentos quando determinada por metodologia tradicional, entretanto, por ser fortemente afetada por níveis de consumo, não é uma boa medida quando determinada por metodologias baseadas em alimentação forçada; 2- a quantidade de alimento consumido afeta tanto a energia metabolizável aparente quanto a energia metabolizável verdadeira na metodologia de alimentação forçada; 3- a energia metabolizável verdadeira corrigida pelo nitrogênio foi a 


\section{Borges et al.}

medida energética menos afetada por metodologias, parecendo ser a melhor forma de estimar o conteúdo energético dos alimentos para aves; 4- a excreção protéica e o balanço de nitrogênio são afetados pelo nível de ingestão, desse modo o menor consumo pela metodologia de alimentação forçada pode levar a maiores erros experimentais; 5- a técnica de alimentação forçada é mais rápida, entretanto, é mais sujeita a erros devido ao baixo conteúdo de alimento ingerido, enquanto que a técnica tradicional parece ser mais precisa na estimativa de energia metabolizável para aves, apresentando menor influência da energia fecal metabólica e urinária endógena; 6- as relações EMV/EMA e EMV $\mathrm{n}_{\mathrm{n}} / \mathrm{EMA}_{\mathrm{n}}$ mostraram-se mais estreitas nos tratamentos com consumo à vontade, significando que os efeitos das perdas endógenas e metabólicas são menos significativos nessa metodologia.

\section{REFERÊNCIAS BIBLIOGRÁFICAS}

ALBINO, L.F.T.; ROSTAGNO, H.S.; FONSECA, J.B et al. Tabela de composição de alimentos concentrados. V. Valores de composição química e de energia determinados em aves de diferentes idades. Rev. Soc. Bras. Zootec., v.10, p.132-146, 1981.

ALBINO, L.F.T.; DE BRUM, P.A.R.; FILHO, F.B. et al. Analise individual versus "pool" de excreta na determinação da energia bruta em ensaio de energia metabolizável. Pesq. Agrop. Bras., v.29, p.467-473, 1994.

ALBINO, L.F.T. Determinação de valores de energia metabolizável e triptofano de alguns alimentos para aves em diferentes idades. 1980. 55p. Dissertação (Mestrado) Universidade Federal de Viçosa, Viçosa, MG

ALBINO, L.F.T. Sistemas de avaliação nutricional de alimentos e suas aplicações na formulação de rações para frangos de corte. 1991. 141p. Tese (Doutorado) Universidade Federal de Viçosa, Viçosa, MG

CHARALAMBOUS, K.; DAGHIR, N.J. Factors affecting the metabolizable energy values of four different poultry feedstuffs. Poult. Sci., v.55, p.1657-1662, 1976.

COLNAGO, G.L.; COSTA, P.M.A.; FONSECA, J.B. et al. Composição química e energia metabolizável de alguns alimentos para poedeiras. Rev. Soc. Bras. Zootec., v.8, p.654-664, 1979.

CUNNIFF, P. (Ed.) Official methods of analysis of AOAC International. 16.ed. Arlington: AOAC International, 1995. v.1.

DALE, N.; FULLER, H.L. Correlation of protein content of feedstuffs with the magnitude of nitrogen correction in true metabolizable energy determinations. Poult. Sci., v.63, p.1008-1012, 1984.

FLORES, M.P.; CASTANON, J.I.R. Effect of level of feed input on true metabolizable energy values and their additivity. Poult. Sci., v.70, p.1381-1385, 1991.

HÄRTEL, H. Influence of food input and procedure of determination on metabolizable energy and digestibility of a diet measured with young and adult birds. Br. Poult. Sci., v.27, p.11-39, 1986.

HAYES, J.P.; DU PREEZ, J.J.; DUCKITT, J.S. et al. Effect of caecectomy on true metabolizable energy and lysine availability in roosters. South Afr. J. Anim. Sci., v.20, p.252-255, 1990.

LIKUSKI, H.J.A.; DORRELL, H.G. A bioassay for rapid determinations of amino acid availability values. Poult. Sci., v.57, p.1658-1660, 1978.

LIMA, I.L.; SILVA, D.J.; ROSTAGNO, H.S. et al. Composição química e valores energéticos de alguns alimentos determinados com pintos e galos, utilizando duas metodologias. Rev. Soc. Bras. Zootec., v.18, p.546-557, 1989.

McNAB, J.M. Factors affecting the energy value of wheat for poultry. World's Poult. Sci. J., v.52, p.69-73, 1996.

MITTELSTAEDT, C.; TEETER, R.G. An evaluation of soft and hard red winter wheat for nitrogen-corrected true metabolizable energy, crude protein, and amino acid content. Poult. Sci., v.72, p.1379-1382, 1993.

MURAMATSU, T.; NAKAJIMA, S.; OKUMURA, J. Modification of energy metabolism by the presence of the gut microflora in the chicken. Br. J. Nutr., v.71, p.709-717, 1994.

NUTRIENT requirements of poultry. 8 ed. Washington: National Academy Press, 1994. 155p.

PUPA, J.M.R.; LEÃO, M.I.; CARVALHO, A.U. et al. Cecectomia em galos sob anestesia local e incisão abdominal. Arq. Bras. Med. Vet. Zootec,. v.50, p.531-535,1998

ROSTAGNO, H.S.; SILVA, D.J.; COSTA, P.M.A. et al. Composição de alimentos e exigências nutricionais de aves e suínos. Viçosa: Imprensa Universitária, 19.ed., 1985. 59p. 
Comparação de métodos de avaliação dos valores energéticos...

SCHANG, M.J. Valor nutritivo de ingredientes y raciones para aves: energia disponible. Rev. Arg. Prod. Anim., v.7, p.599608, 1987.

SHIRES, A.; ROBBLEE, A.R.; HARDIN, R.T. et al. Effect of the age of chickens on the true metabolizable energy values of feed ingredients. Poult. Sci., v.59, p.396-403, 1980.

SIBBALD, I.R. A bioassay for true metabolizable energy in feedingstuffs. Poult. Sci., v.55, p.303-308, 1976

SIBBALD, I.R.; MORSE, P.M. Effects of the nitrogen correction and feed intake on true metabolizable energy values. Poult. Sci., v.61, p.138-142, 1982

SIBBALD, I.R.; WOLYNETZ, M.S. Comparisons of bioassays for true metabolizable energy adjusted to zero nitrogen balance. Poult. Sci., v.67, p.1192-1202, 1988.

SIBBALD, I.R.; WOLYNETZ, M.S. Estimates of retained nitrogen used to correct estimates of bioavailable energy. Poult. Sci., v.64, p.1506-1513, 1985. 